\title{
Use and cost of hospitalization in dementia: longitudinal results from a community-based study
}

\author{
Carolyn W. Zhu ${ }^{1,2}$, Stephanie Cosentino ${ }^{3}$, Katherine Ornstein ${ }^{4}$, Yian $\mathrm{Gu}^{3}$, Howard Andrews ${ }^{5}$ and Yaakov Stern ${ }^{3}$ \\ ${ }^{1}$ Department of Geriatrics and Palliative Medicine, Icahn School of Medicine at Mount Sinai, New York, NY, USA \\ ${ }^{2}$ James J Peters VA Medical Center, Bronx, NY, USA \\ ${ }^{3}$ Cognitive Neuroscience Division of the Gertrude H. Sergievsky Center, Taub Institute for Research on Alzheimer's Disease and the Aging \\ Brain, and Department of Neurology, Columbia University Medical Center, New York, NY, USA \\ ${ }^{4}$ The Samuel Bronfman Department of Medicine, Division of General Internal Medicine, Icahn School of Medicine at Mount Sinai, \\ New York, NY, USA \\ ${ }^{5}$ Department of Biostatistics, Mailman School of Public Health, Columbia University, New York, NY, USA \\ Correspondence to: Carolyn W. Zhu, PhD, E-mail: carolyn.zhu@mssm.edu
}

\begin{abstract}
Objectives: The aim of this study is to examine the relative contribution of functional impairment and cognitive deficits on risk of hospitalization and costs.

Methods: A prospective cohort of Medicare beneficiaries aged 65 and older who participated in the Washington Heights-Inwood Columbia Aging Project (WHICAP) were followed approximately every 18 months for over 10 years (1805 never diagnosed with dementia during study period, 221 diagnosed with dementia at enrollment). Hospitalization and Medicare expenditures data (1999-2010) were obtained from Medicare claims. Multivariate analyses were conducted to examine (1) risk of all-cause hospitalizations, (2) hospitalizations from ambulatory care sensitive (ACSs) conditions, (3) hospital length of stay (LOS), and (4) Medicare expenditures. Propensity score matching methods were used to reduce observed differences between demented and non-demented groups at study enrollment. Analyses took into account repeated observations within each individual.
\end{abstract}

Results: Compared to propensity-matched individuals without dementia, individuals with dementia had significantly higher risk for all-cause hospitalization, longer LOS, and higher Medicare expenditures. Functional and cognitive deficits were significantly associated with higher risks for hospitalizations, hospital LOS, and Medicare expenditures. Functional and cognitive deficits were associated with higher risks of for some ACS but not all admissions.

Conclusions: These results allow us to differentiate the impact of functional and cognitive deficits on hospitalizations. To develop strategies to reduce hospitalizations and expenditures, better understanding of which types of hospitalizations and which disease characteristics impact these outcomes will be critical. Copyright (C) 2014 John Wiley \& Sons, Ltd.

Key words: dementia; hospitalization; healthcare expenditures; longitudinal follow-up History: Received 26 June 2014; Accepted 9 September 2014; Published online in Wiley Online Library (wileyonlinelibrary.com) DOI: 10.1002 /gps.4222

\section{Introduction}

Hospitalizations are the largest component of total healthcare expenditures, accounting for almost half (47\%) of all Medicare fee-for-service (FFS) expenditures in 2012 (Lin et al., 2013). In individuals with dementia, the proportion of Medicare expenditures for inpatient hospitalization is even higher (Albert et al.,
1999; Landi et al., 1999; Lyketsos et al., 2000; Fillenbaum et al., 2001; Andrieu et al., 2002; Bynum et al., 2004; Natalwala et al., 2008; Tuppin et al., 2009; Zekry et al., 2009; Ehlenbach et al., 2010; Guijarro et al., 2010; Rudolph et al., 2010; Phelan et al., 2012; Alzheimer's Association, 2013; MedPAC (Medicare Payment Advisory Commission), June 2013; Schneider et al., 2013). Most studies showed 
individuals with dementia at higher rates of hospitalizations than non-demented individuals, with higher healthcare expenditures (Lyketsos et al., 2000; Bynum et al., 2004; Zhao et al., 2008; Phelan et al., 2012; Lin et al., 2013). Reducing hospitalizations and related costs is an important need in bringing down the cost of dementia care. Some hospitalizations are necessary and expected; others indicate suboptimal ambulatory care and are potentially avoidable. A recent study using data from an integrated healthcare system reported incident dementia was significantly associated with increased risk of all-cause hospitalizations and for across all ACS admissions (Phelan et al., 2012). Another study based on Medicare claims reported more mixed results. Beneficiaries with dementia were found to be at higher risks of hospitalization for several ACS conditions (e.g. diabetes, hypertension) but at lower risks for hospitalization for others (e.g. chronic obstructive pulmonary disease (COPD)/ asthma and congestive heart failure (CHF)) (Lin et al., 2013). Among beneficiaries with hospitalizations for ACS conditions, total Medicare expenditures were higher for individuals with dementia than those without dementia.

While it is clear that dementia increases hospitalizations and costs, which aspects of the disease may be the main contributing factors are unclear. For example, the relative contribution to increases in the risks for hospitalizations and expenditures from cognitive or functional impairment remains unclear. These distinctions are important, because efforts to reduce hospitalizations and related expenditures would differ depending on their sources.

With few exceptions, existing studies have used Medicare claims to identify dementia and have relatively short follow-up time. Claims-based diagnoses have little information on disease severity and have been shown to under-estimate or mis-identify dementia, often identifying dementia at later stages (Newcomer et al., 1999; Fillit et al., 2002; Lin et al., 2010). In this study, we take advantage of a unique longitudinal study that prospectively followed a cohort of Medicare beneficiaries for whom comprehensive cognitive and functional assessments were systematically and frequently carried out, yielding accurate in-person diagnoses of dementia, and for whom data on hospitalizations are available from Medicare claims. Methodologically, characteristics of subjects with and without dementia often differ substantially at study enrollment, and these differences may confound estimated effects of disease characteristics on outcomes. We used propensity score methods to generate more balanced groups to increase confidence in our results.

\section{Methods}

Participants

Participants were recruited from the Washington Heights-Inwood Columbia Aging Project (WHICAP), a multiethnic, population-based, prospective study of cognitive aging in Medicare beneficiaries aged 65 and older residing in a geographically defined area of northern Manhattan. Lists of all Medicare or Medicaid recipients in the study area were obtained from Health Care Financing Administration (HCFA, since 2001, Centers for Medicare and Medicaid Services, CMS). Potential subjects were then drawn by stratified random sampling into one of six strata based on age (65-74, 75+) and ethnicity (Hispanics, non-Hispanic blacks, non-Hispanic whites). All individuals were sent a letter from HCFA explaining that they had been selected to participate in a study of aging by investigators at Columbia University. An initial cohort of 2125 subjects was recruited during 1992-1994. A second cohort of 2183 subjects was formed during 19992002 from new lists of beneficiaries obtained from HCFA using similar methods. Detailed descriptions of study methodology have been reported previously (Tang et al., 1998).

At the time of study entry, each subject underwent an in-person interview of general health and functional ability followed by a standardized assessment which included medical history, physical and neurological examination, and a neuropsychological battery. After baseline assessment, subjects were followed at approximately 18 -month intervals until death or drop out. Evaluations were conducted in either English or Spanish, based on participant's primary language or preference. Recruitment, informed consent and study procedures were approved by the Institutional Review Boards of Columbia Presbyterian Medical Center, New York State Psychiatric Institute, and CMS Privacy Board. Written informed consent was obtained from all subjects.

\section{Diagnosis of dementia}

At baseline and each follow-up, assessment of dementia was made at diagnostic conferences attended by a group of neurologists, psychiatrists, and neuropsychologists, using results from the neuropsychological battery and evidence of impairment in social or occupational function (McKhann et al., 1984; Stern et al., 1992). The type of dementia was subsequently determined based on Diagnostic and Statistical Manual of Mental Disorders, Revised Fourth Edition criteria. 
Diagnosis of probable or possible AD was made based on criteria outlined by the National Institute of Neurological and Communicative Disorders and Stroke (NINCDS)_Alzheimer's Disease and Related Disorders Association (McKhann et al., 1984). These criteria and diagnostic methods have been used extensively in the literature. Because of the epidemiologic nature of the study, subjects' primary care providers were not notified of a study diagnosis of dementia.

\section{Derivation of the study sample}

We used a retrospective cohort design to examine hospitalizations in WHICAP subjects. Using social security number and Medicare beneficiary ID, 2476 subjects were matched to the Medicare Beneficiary Summary file from 1 January 1999 to 31 December 2010 and not enrolled in a Medicare HMO. Of these 2476 subjects, 93 (3.7\%) subjects did not have any Medicare utilization any time during the study and were excluded from the analyses. Of the 2383 individuals who were followed from WHICAP enrollment or beginning of Medicare data availability, to end of study or death, 1805 (75.7\%) were never diagnosed with dementia (never demented cases), 221 (9.3\%) were diagnosed with dementia at study enrollment (prevalent cases), and $357(15.0 \%)$ were nondemented at study entry but developed dementia at some point during the study (follow-up period $=8.8 \pm 4.4$ years, $4.6 \pm 2.6$ years since dementia onset). This study focuses on the 2026 prevalent and never demented cases.

\section{Variables}

Outcome measures. Hospitalization data were obtained using Medicare Provider and Analysis Review (MedPAR) Files. We examined four sets of outcomes: (1) hospitalization requiring at least one overnight stay; (2) length of stay (LOS); (3) Medicare expenditure for the stay; and (4) ACS admissions. We used the Prevention Quality Indicators (PQIs) developed by the Agency for Healthcare Research and Quality (AHRQ) to identify the following ACS conditions:(Agency for Healthcare Research and Quality, 2001) diabetes mellitus, COPD, CHF, bacterial pneumonia, urinary tract infection (UTI), hypertension, dehydration, angina, and asthma. Medicare expenditures were reported in constant 2010\$ using the medical care component of the Consumer Price Index (Bureau of Labor Statistics, 2012).

Dementia severity. To obtain a more complete understanding of which aspects of dementia severity were associated with increased hospitalizations and expenditure, subjects' cognitive and functional deficits were measured as follows. Cognition was assessed across the following five domains in a structured in-person assessment: memory, abstract reasoning, visual-spatial, language, and executive-speed. Z-scores for individual and domain tests were computed using scores from non-demented controls in WHICAP with a similar distribution of age, education, and ethnicity to dementia patients. A composite cognitive score (CS) was derived by averaging domain scores. Detailed construction of the CS score has been reported earlier (Cosentino et al., 2008).

Function was assessed at each interval using the Blessed Dementia Rating Scale to ascertain difficulties performing various activities of daily living for nonphysical reasons (range $=0-13$ ) (Blessed et al., 1968). Higher scores indicate greater functional impairment.

Other covariates. The following potential confounders were included a priori in multivariate analyses based on known associations between these predictors and risks of hospitalization: socio-demographic characteristics (age, sex, ethnicity, and years of education), comorbid conditions, depressive symptoms, and nursing home residence. Because death is strongly associated with hospitalizations and expenditures, we included an indicator for death during the study period. Ethnicity was based on self-report using 1990 US Census format and grouped into non-Hispanic white, non-Hispanic black, Hispanic, or other. Education was dichotomized at median ( $\leq 8$ vs. $>8$ years). Comorbid conditions were assessed using a modified Charlson Index of Comorbidity which included myocardial infarct, CHF, peripheral vascular disease, hypertension, COPD, arthritis, gastrointestinal disease, mild liver disease, diabetes, chronic renal disease, and systemic malignancy. All items received weights of one, with the exception of chronic renal disease and systemic malignancy, which were weighted two (Charlson et al., 2008). Current depressive symptoms were assessed with the 10-item version of the Center for Epidemiologic Studies-Depression (CES-D) scale (Irwin et al., 1999). The conventional cutoff score of $\geq 4$ was used to indicate the presence of depressive symptoms. To assess secular trend over time, an indicator for follow-up year was included.

\section{Analysis}

Our analyses proceeded in two steps. First, because characteristics that may have confounding effects on outcomes differ substantially between subjects in prevalent and never demented groups, we used propensity 
score methods to generate more balanced groups that have similar observed characteristics. Propensity scores are conditional probabilities of belonging to a particular group, given a set of observed background characteristics (D’Agostino, 1998; Austin, 2008). Because subjects were followed over time, propensity score matching was conducted at study enrollment. We used an expansive definition of balancing characteristics as suggested by Rubin and included age, gender, race/ethnicity, education, Medicaid eligibility, comorbid conditions, living arrangement, and year in our propensity score matching model (Rubin, 2007). Clinical characteristics that are specifically related to dementia (i.e. BDRS, CS) were excluded.

Using the propensity-matched sample, we then constructed a longitudinal data set of hospitalization during each assessment interval. With this longitudinal data set, logistic regressions were used to examine risks for all-cause and ACS admissions. Among subjects who had at least one hospitalization, truncated negative binomial regressions were used to estimate LOS (Long and Freese, 2006). Incidence rate ratios (IRR) for the LOS models were reported as estimates of the proportional increase (for values greater than 1 ) or decrease (for values between 0 and 1) of LOS that was associated with a one unit increase in an explanatory variable (Hilbe, 2011). Generalized linear models with logarithmic cost as the dependent variable were used to estimate Medicare expenditures. Coefficients estimated proportional change in Medicare expenditures for each unit change in the explanatory variable. Because subjects were followed over time, cluster robust standard errors were estimated for all models to account for multiple observations within individuals. Improved fit of the final models was established by reduction in Bayesian Information Criterion (BIC). All analyses were performed using Stata 11.0. Analyses of risks of all-cause hospitalization included the full sample. Analyses of LOS, ACS admissions, and Medicare expenditures included individuals with at least one hospitalization.

We conducted the following additional sensitivity analyses: (1) we excluded individuals observed for less than one year and (2) we excluded individuals who died during the study. Results from these sensitivity analyses were similar to results from the primary models.

\section{Results}

Unmatched sample characteristics at study enrollment

In the unmatched sample, demographic and health characteristics of the prevalent and never demented groups differed significantly at study enrollment (Table 1).
Compared to those never diagnosed with dementia, subjects with dementia were older $(84 \pm 7.7$ vs. $77 \pm 6.5$ years), more likely to be female ( $68 \%$ vs. $66 \%$ ), Hispanic ( $58 \%$ vs. $41 \%$ ), widowed ( $51 \%$ vs. $35 \%$ ), and living with an adult child (19\% vs. $12 \%)$ or another person (17\% vs. $12 \%)$. They also had fewer years of schooling $(6.5 \pm 4.3$ vs. $10.6 \pm 4.8$ years) and were more likely to have Medicaid (57\% vs. $34 \%)$. At study enrollment, almost all subjects who were never-demented were living at home (99\%), compared to $96 \%$ of those with dementia. Twothirds of those with dementia died during the course of study follow-up, but only one-third (34\%) of never demented died. On average, subjects with dementia were followed for 3 years compared to 4.6 years for nondemented subjects. Number of chronic conditions was similar between prevalent and never demented groups $(2.8 \pm 2.0$ vs. $2.6 \pm 2.0)$. The most common conditions included hypertension (64\%), arthritis (53\%), and diabetes $(21 \%)$. Compared to those never diagnosed with dementia, subjects with dementia were more likely to have had a stroke ( $17.6 \%$ vs. $9.2 \%)$, CHF (9.5\% vs. $4.6 \%)$, and angina $(3.2 \%$ vs. $0.8 \%)$, but less likely to have cancer (6.3\% vs. $14.5 \%)$.

At study enrollment, most subjects who were never diagnosed with dementia had a CDR of $0(78.5 \%)$, the rest had a CDR of $0.5(21.3 \%)$. Most of the subjects with dementia had a CDR of $1(70 \%)$, consistent with mild dementia, the rest were almost evenly distributed between CDR of $2(15.8 \%)$, and CDR of 3 (14.0\%). Those with dementia had substantially worse scores in function and cognition than the never demented subjects. All cognitive scores in the dementia group were more than 1.1 $\mathrm{SD}$ below normative non-demented controls.

In the unmatched sample, hospitalizations and related expenditures of demented and never demented groups differed significantly during the year of study enrollment. Compared to $19.6 \%$ of non-demented subjects, $30.3 \%$ of subjects with dementia had at least one hospitalization. Subjects with dementia also had higher number of hospitalizations ( $1.9 \pm 1.3$ vs. $1.6 \pm 1.1)$, higher number of days of hospitalizations $(13.8 \pm 15.9$ vs. $10.4 \pm 12.0)$, and higher hospitalization expenditures $(\$ 24,123 \pm 30,837$ vs. $\$ 19,649 \pm 20,691)$. Most subjects with dementia had at least one ACS admission (83.5\%) as did nondemented subjects (79.3\%). All ACS admission rates were higher for subjects with dementia.

\section{Propensity matching}

Using propensity score matching with caliper (caliper width of .25 of standard deviation of the logit of the propensity scores), 171 dementia subjects were 


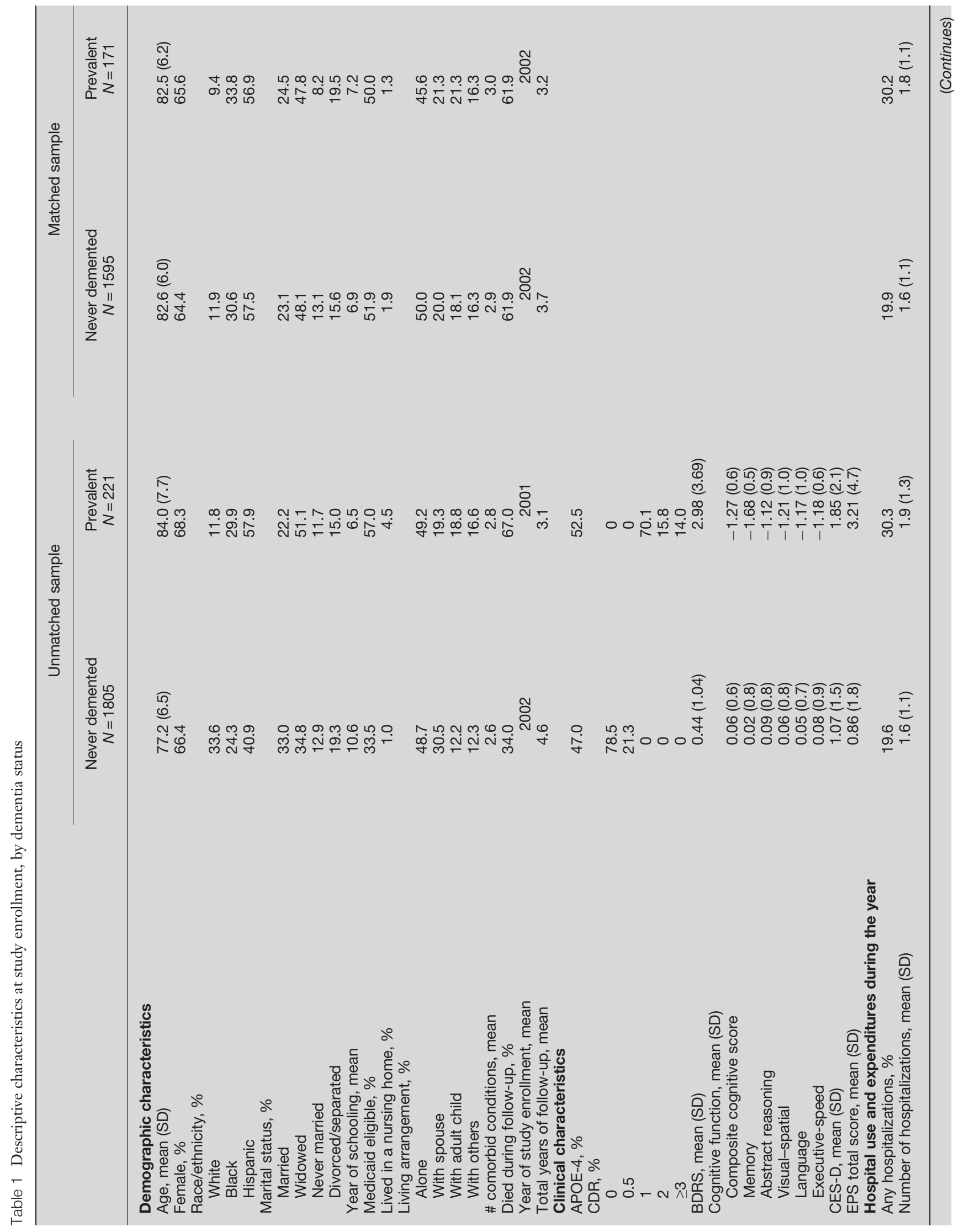




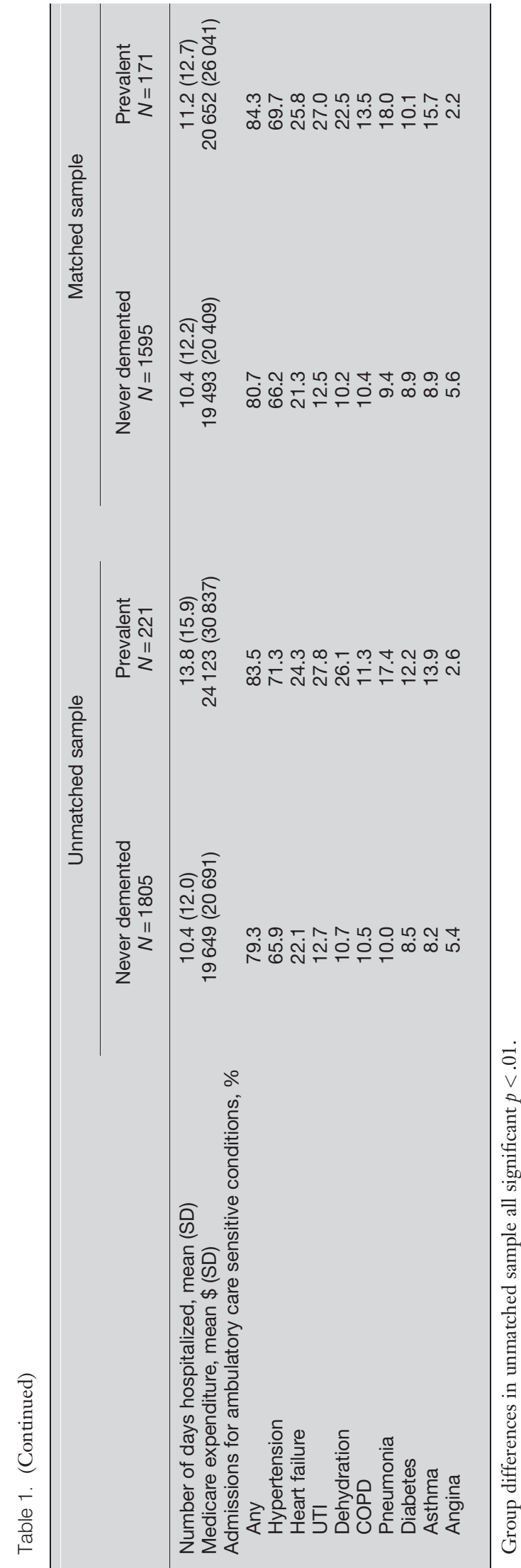

matched to 1595 never demented subjects. Between group differences in unmatched samples were substantially reduced after propensity-score matching (Table 1). Standardized differences on all measured variables in the matched sample were $<9$, suggesting that the two groups were well-matched on these characteristics (Austin, 2008).

Associations with risks of all-cause hospitalization, hospital length of stay, and medicare expenditures

Table 2 first confirms what is known about dementia, though mostly in unmatched samples. Compared to propensity matched subjects without dementia, subjects with clinically diagnosed dementia had higher risk of hospitalization $(\mathrm{OR}=1.462,95 \%$ confidence interval $[\mathrm{CI}]=[1.244,1.942])$, longer hospital LOS $(\mathrm{IRR}=1.059,95 \% \mathrm{CI}=[0.998,1.129])$, and $18 \%$ higher Medicare expenditures $(95 \% \mathrm{CI}=[0.022,0.410])$. Focusing on subjects' functional and cognitive deficits, subjects' functional deficits were significantly associated with higher risks of hospitalization, longer LOS, and higher Medicare expenditures. Subjects' cognitive deficits also were significantly associated with higher risks of hospitalization and longer LOS, but were not associated with Medicare expenditures.

\section{Associations with risks of hospitalizations for ambulatory care sensitive conditions}

In terms of ACS hospitalizations, compared to propensity matched subjects without dementia, subjects with dementia had higher risk of hospitalizations for UTI $(\mathrm{OR}=2.111,95 \% \mathrm{CI}=[1.224,3.641])$ and dehydration $(\mathrm{OR}=2.390, \quad 95 \% \quad \mathrm{CI}=[1.404,4.070])$ (Table 3). Subjects' functional deficits were associated with increased risk of hospitalizations for diabetes, UTI, and dehydration, but decreased risk of hospitalizations for hypertension. Better cognitive score was associated with decreased risk of hospitalizations for diabetes, pneumonia, and asthma, and marginally for hypertension.

\section{Discussion}

In this study we followed for more than 10 years a cohort of individuals with dementia along with a comparison cohort of individuals who were never diagnosed with dementia during the study and examined differences in hospitalization-related outcomes over time. Results showed significantly higher risks 
Table 2 Effects of dementia severity on risk of hospitalization, hospital LOS and Medicare Expenditures in a propensity matched sample

\begin{tabular}{lccc}
\hline & Hospitalization & Hospital length of stay & Medicare Expenditure \\
\cline { 2 - 4 } & OR/SE & IRR/SE & b/SE \\
\hline $\begin{array}{l}\text { Model A } \\
\begin{array}{l}\text { Presence of dementia diagnosis } \\
\text { Model B }\end{array}\end{array}$ & $1.462(0.330)^{\star}$ & $1.059(0.035)^{+}$ & $0.181(0.101)^{\star}$ \\
$\begin{array}{l}\text { BDRS score } \\
\text { CS score }\end{array}$ & $1.274(0.059)^{\star *}$ & $1.031(0.007)^{\star *}$ & $0.048(0.017)^{\star *}$ \\
& $0.761(0.092)^{*}$ & $0.947(0.020)^{\star *}$ & $0.042(0.055)$ \\
\hline
\end{tabular}

${ }^{+} p<.10,{ }^{*} p<.05,{ }^{* *} p<.01$

Each model controlled for age, sex, ethnicity, years of education, comorbid conditions, depressive symptoms, living arrangement, death during the study period, and follow-up year. Cluster robust standard errors were reported.

for all-cause hospitalizations, hospital LOS, and Medicare expenditures in individuals with dementia compared to their propensity-matched counterparts without dementia. Differentiating the impact of functional and cognitive deficits on hospitalizations, we found that functional and cognitive deficits both were significantly associated with higher risks for hospitalizations, hospital LOS, and Medicare expenditures.

Compared to propensity matched individuals without dementia, results showed that individuals with dementia had significantly higher risks of hospitalization for UTI and dehydrations, but not for other conditions, suggesting suboptimal outpatient care in these conditions but not others. Worse functional status was associated with higher risks of hospitalization for diabetes and UTI, marginally higher for dehydration, but lower for hypertension. Worse cognitive status was associated with higher risks of hospitalizations for diabetes, pneumonia, hypertension, and dehydration. Why do we observe these differences? It is possible that differences in the nature of these conditions, routes by which individuals were admitted to the hospital, or other factors may be more or less susceptible to cognitive or functional deficits. In an early study on acute hospitalization in Alzheimer's patients, dependence in bathing, but not other functional deficits, nor cognitive deficits, was found to be a significant predictor of hospitalizations (Andrieu et al., 2002). Although the authors did not differentiate reasons for hospitalizations, it is possible that dependence in bathing was more problematic for certain conditions than other conditions. Future studies clarifying the reasons for these differences are needed in order to develop strategies to prevent unnecessary hospitalization.

Our study adds to a small but increasing set of studies that have examined the relationship between dementia and hospitalizations. Among others, differences in sample characteristics, method of identifying dementia patients, study design, and methodology may explain some of the differences in results. Several differences are of particular note. A recent analysis using data from an integrated healthcare system reported that incident dementia was significantly associated with increased risk of all-cause hospitalizations and for hospitalizations across all ACS conditions (Phelan et al., 2012). But another study using a propensity matching method but with Medicare claims data found higher risks of hospitalization for only certain conditions (Lin et al., 2013). Individuals in these samples may have very different patterns of healthcare utilization. Compared to individuals who were enrolled in one integrated healthcare system, our sample is much older, poorer and sicker, and more ethnically and culturally diverse and may already have less than optimal care. It is possible that the less than optimal care may be masking any additional effect of dementia on ACS hospitalizations. It should also be noted that hospitalizations for hypertension is unusually high in our sample (Blustein et al., 1998; Agency for Healthcare Research and Quality, 2001). More work clearly is needed to better understand factors that may have contributed to the high rates of ACS hospitalization and particularly for hypertension.

Our study has some important strengths. It represents a unique large scale epidemiologic study examining the risk factors for hospitalization outcomes in a racial/ethnically diverse, vulnerable population. With few exceptions, most existing studies have used Medicare claims to identify dementia and with short followup time to assess hospitalizations. Use of claims data may lead to mis-identification or delayed identification of dementia, with little information on disease severity. We overcome these issues by supplementing Medicare claims data with clinical data obtained from frequent and careful assessment and long follow-up. The careful identification of disease characteristics along with use 


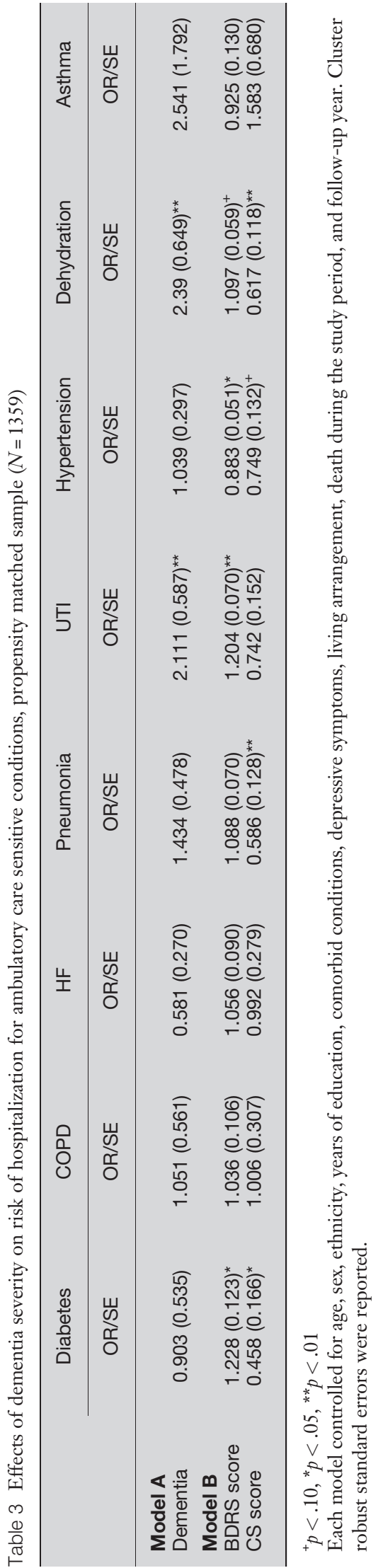

of claims allows a comprehensive picture of hospitalization and adjustment of confounders. Specifically, looking at function and cognition separately allows assessment of effects on hospitalization specific to these aspects of dementia. Using propensity score matched samples that reduced the large differences between demented and non-demented cohorts, confidence in our results is increased that the estimated differences in the outcomes are due to characteristics of dementia and not from other factors that could influence the outcomes. Results from this study call for greater attention on cognitive and functional status and not just dementia diagnosis in predicting hospitalization outcomes. As hospitals develop strategies to reduce hospitalization and expenditures, better understanding of which types of hospitalizations and which patient factors impact these outcomes will be critical.

\section{Conflict of interest}

None declared.

\section{Key points}

- Individuals with dementia had significantly higher risk for all-cause hospitalization, longer LOS and higher Medicare expenditures. Functional and cognitive deficits both were significantly associated with higher risks for hospitalizations, hospital LOS, and Medicare expenditures.

- Functional and cognitive deficits were associated with higher risks of for admissions for some but not all ambulatory care sensitive conditions.

- Better understanding of which types of hospitalizations and which disease characteristics impact hospitalization outcomes will be critical to develop strategies to reduce hospitalizations and expenditures.

\section{Acknowledgements}

This research was supported by grants from the National Institute on Aging (AG07370, AG037212).

\section{Author contributions}

Drs. Zhu and Stern had full access to all of the data in the study and take responsibility for the integrity of 
the data and the accuracy of the data analysis. Study concept and design: Zhu, Stern; acquisition of data: Stern, Howard, Zhu; Analysis and interpretation of data: Zhu, Cosentino, Ornstein, Gu, Andrews, Stern; drafting of the manuscript: Zhu; critical revision of the manuscript for important intellectual content: Cosentino, Ornstein, Gu, Andrews, Stern; statistical analysis: Zhu; obtaining funding: Stern, Zhu; Administrative, technical, and material support: Cosentino, Ornstein, $\mathrm{Gu}$, Andrews.

\section{References}

Agency for Healthcare Research and Quality. 2001. AHRQ quality indicators—guide to prevention quality indicators: hospital admission for ambulatory care sensitive conditions. Agency for Healthcare Research and Quality: Rockville, MD.

Albert SM, Costa R, Merchant C, et al. 1999. Hospitalization and Alzheimer's disease: results from a community-based study. J Gerontol A Biol Sci Med Sci 54: M267-M271. Alzheimer's Association. 2013. Alzheimer's Disease Facts and Figures.

Andrieu S, Reynish E, Nourhashemi F, et al. 2002. Predictive factors of acute hospitalization in 134 patients with Alzheimer's disease: a one year prospective study. Int J Geriatr Psychiatry 17: 422-426.

Austin PC. 2008. A critical appraisal of propensity-score matching in the medical literature between 1996 and 2003. Stat Med 27: 2037-2049.

Blessed G, Tomlinson BE, Roth M. 1968. The association between quantitative measures of dementia and of senile change in the cerebral grey matter of elderly subjects. Br J Psychiatry 114: 797-811.

Blustein J, Hanson K, Shea S. 1998. Preventable hospitalizations and socioeconomic status. Health Aff (Millwood) 17: 177-189.

Bureau of Labor Statistics. 2012. Consumer price index. Available at: http://www.bls. gov/cpi/home.htm (accessed 10 March 2014).

Bynum JP, Rabins PV, Weller W, et al. 2004. The relationship between a dementia diagnosis, chronic illness, Medicare expenditures, and hospital use. J Am Geriatr Soc 52: 187-194.

Charlson ME, Charlson RE, Peterson JC, et al. 2008. The Charlson comorbidity index is adapted to predict costs of chronic disease in primary care patients. J Clin Epidemiol 61: 1234-1240.

Cosentino S, Scarmeas N, Helzner E, et al. 2008. APOE epsilon 4 allele predicts faster cognitive decline in mild Alzheimer disease. Neurology 70: 1842-1849.

D'Agostino RB Jr. 1998. Propensity score methods for bias reduction in the comparison of a treatment to a non-randomized control group. Stat Med 17: 2265-2281.

Ehlenbach WJ, Hough CL, Crane PK, et al. 2010. Association between acute care and critical illness hospitalization and cognitive function in older adults. JAMA 303: 763-770.

Fillenbaum G, Heyman A, Peterson BL, Pieper CF, Weiman AL. 2001. Use and cost of hospitalization of patients with $\mathrm{AD}$ by stage and living arrangement: CERAD XXI. Neurology 56: 201-206.

Fillit H, Geldmacher DS, Welter RT, Maslow K, Fraser M. 2002. Optimizing coding and reimbursement to improve management of Alzheimer's disease and related dementias. J Am Geriatr Soc 50: 1871-1878.
Guijarro R, San Roman CM, Gomez-Huelgas R, et al. 2010. Impact of dementia on hospitalization. Neuroepidemiology 35: 101-108.

Hilbe J. 2011. Negative binomial regression. Cambridge University Press: Cambridge. Irwin M, Artin KH, Oxman MN. 1999. Screening for depression in the older adult: criterion validity of the 10-item Center for Epidemiological Studies Depression Scale (CES-D). Arch Intern Med 159: 1701-1704.

Landi F, Gambassi G, Lapane KL, et al. 1999. Impact of the type and severity of dementia on hospitalization and survival of the elderly. The SAGE Study Group. Dement Geriatr Cogn Disord 10: 121-129.

Lin PJ, Fillit HM, Cohen JT, Neumann PJ. 2013. Potentially avoidable hospitalizations among Medicare beneficiaries with Alzheimer's disease and related disorders. Alzheimers Dement 9: 30-38.

Lin PJ, Kaufer DI, Maciejewski ML, et al. 2010. An examination of Alzheimer's disease case definitions using Medicare claims and survey data. Alzheimers Dement 6: 334-341.

Long J, Freese J. 2006. Regression Models for Categorical Dependent Variables Using Stata. Stata Press: College Station, TX.

Lyketsos CG, Sheppard JM, Rabins PV. 2000. Dementia in elderly persons in a general hospital. Am J Psychiatry 157: 704-707.

Mckhann G, Drachman D, Folstein M, et al. 1984. Clinical diagnosis of Alzheimer's disease: report of the NINCDS-ADRDA Work Group under the auspices of Department of Health and Human Services Task Force on Alzheimer's Disease. Neurology 34: 939-944.

MedPAC (Medicare Payment Advisory Commission). June 2013. A Data Book: Health Care Spending and the Medicare Program. Washington DC.

Natalwala A, Potluri R, Uppal H, Heun R. 2008. Reasons for hospital admissions in dementia patients in Birmingham, UK, during 2002-2007. Dement Geriatr Cogn Disord 26: 499-505.

Newcomer R, Clay T, Luxenberg JS, Miller RH. 1999. Misclassification and selection bias when identifying Alzheimer's disease solely from Medicare claims records. J Am Geriatr Soc 47: 215-219.

Phelan EA, Borson S, Grothaus L, Balch S, Larson EB. 2012. Association of incident dementia with hospitalizations. JAMA 307: 165-172.

Rubin DB. 2007. The design versus the analysis of observational studies for causal effects: parallels with the design of randomized trials. Stat Med 26: 20-36.

Rudolph JL, Zanin NM, Jones RN, et al. 2010. Hospitalization in communitydwelling persons with Alzheimer's disease: frequency and causes. J Am Geriatr Soc 58: 1542-1548.

Schneider AL, Gottesman RF, Mosley T, et al. 2013. Cognition and incident dementia hospitalization: results from the atherosclerosis risk in communities study. Neuroepidemiology 40: 117-124.

Stern Y, Andrews H, Pittman J, et al. 1992. Diagnosis of dementia in a heterogeneous population. Development of a neuropsychological paradigm-based diagnosis of dementia and quantified correction for the effects of education. Arch Neurol 49: 453-460.

Tang MX, Stern Y, Marder K, et al. 1998. The APOE-epsilon4 allele and the risk of Alzheimer disease among African Americans, whites, and Hispanics. JAMA 279: 751-755.

Tuppin P, Kusnik-Joinville O, Weill A, Ricordeau P, Allemand H. 2009. Primary health care use and reasons for hospital admissions in dementia patients in france: database study for 2007. Dement Geriatr Cogn Disord 28: 225-232.

Zekry D, Herrmann FR, Grandjean R, et al. 2009. Does dementia predict adverse hospitalization outcomes? A prospective study in aged inpatients. Int J Geriatr Psychiatry 24: 283-291.

Zhao Y, Kuo TC, Weir S, Kramer MS, Ash AS. 2008. Healthcare costs and utilization for Medicare beneficiaries with Alzheimer's. BMC Health Serv Res 8: 108. 